Cite this: Analyst, 2013, 138, 7294

Received 13th August 2013

Accepted 18th October 2013

DOI: $10.1039 /$ c3an01540h

\section{Single-channel electrophysiology of cell-free expressed ion channels by direct incorporation in lipid bilayers $\uparrow$}

\author{
Mark S. Friddin, ${ }^{\text {ab }}$ Natalie P. Smithers, ${ }^{\text {bc }}$ Maïwenn Beaugrand, ${ }^{d}$ Isabelle Marcotte, ${ }^{* d}$ \\ Philip T. F. Williamson, ${ }^{\star b c}$ Hywel Morgan $^{\mathrm{ab}}$ and Maurits R. R. de Planque ${ }^{\star a b}$
}

www.rsc.org/analyst

Single-channel electrophysiology with lipid bilayer systems requires ion channel expression, purification from cell culture, and reconstitution in proteoliposomes for delivery to a planar bilayer. Here we demonstrate that single-channel current measurements of the potassium channels KCSA and hERG $_{\text {S5-56 }}$ can be obtained by direct insertion in interdroplet lipid bilayers from microliters of a cell-free expression medium.

Electrophysiology is the gold standard for the functional characterization of ion channel proteins, including screening of new drugs that target this pharmaceutically important class of membrane proteins., ${ }^{\mathbf{1} 2}$ The patch clamp technique involves intimate contact of an electrode-containing glass pipette with the membrane of a cell in which the channel of interest is overexpressed, and is used most widely for ensemble current measurements of channel populations. The alternative 'bilayer lipid membrane' (BLM) approach, in which purified ion channels are introduced by proteoliposome fusion into a pure-lipid membrane that separates two electrode-containing aqueous compartments, is typically employed for current measurements of single channels. ${ }^{3,4}$ Automated instruments for mediumthroughput patch clamp electrophysiology have entered the market in the last decade, ${ }^{\mathbf{5}, \mathbf{6}}$ whereas systems for automated and/or parallel BLM electrophysiology are under development by several research groups and companies. ${ }^{7-9}$

${ }^{a}$ Electronics and Computer Science, University of Southampton, Southampton, SO17 1BJ, UK. E-mail: mdp@ecs.soton.ac.uk; Fax: +44 (0)23 8059 3029; Tel: +44 (0)23 80599307

${ }^{b}$ Institute for Life Sciences, University of Southampton, Southampton, SO17 1BJ, UK ${ }^{c}$ Centre for Biological Sciences, University of Southampton, Southampton, SO17 1BJ, UK. E-mail: P.T.F.Williamson@soton.ac.uk; Tel: +44 (0)23 80594350

${ }^{d}$ Département de Chimie, Université du Québec à Montréal, GRASP/CQMF, P.O. Box 8888, Downtown Station, Montréal, H3C 3P8, Canada. E-mail: marcotte.isabelle@ uqam.ca; Fax: +1 (0)514 987 4054; Tel: +1 (0)514 9873000 ext. 5015

$\dagger$ Electronic supplementary information (ESI) available: Protein expression methods, expression verification, bilayer current measurements and analysis. See DOI: 10.1039/c3an01540h
Although there are recent developments toward patch clamping of giant proteoliposomes, ${ }^{\mathbf{1 0}}$ patch clamping typically relies on eukaryotic cells that are able to overexpress the ion channel of interest with concomitant cell membrane incorporation. ${ }^{2}$ However, this is not possible when the expressed channel aggregates or when membrane incorporation is toxic to the cell. Proteoliposome formation, for patch clamping or for the BLM method, also requires ion channel overexpression, but, at the cell culture stage, not necessarily as solubilized or membrane-incorporated protein, and is not restricted to eukaryotic cells. ${ }^{3}$ This offers more options to obtain the desired ion channel but at the cost of having to purify it from cell culture, with subsequent reconstitution from detergent solution into liposomes, which is a laborious process that requires a relatively high protein yield.

In recent years, considerable progress has been made with the cell-free expression of membrane proteins, ${ }^{\mathbf{1 1}}$ which bypasses any cytotoxicity problems and facilitates protein purification. Bacterial lysates that contain the ribosomal machinery and are supplemented with amino acids, a metabolic energy supply and a protein-encoding plasmid, have been shown to express a large variety of membrane proteins, either as precipitates or solubilized in detergent micelles. ${ }^{\mathbf{1 2 - 1 4}}$ Interestingly, when liposomes are added to the cell-free reaction mixture, spontaneous reconstitution has been demonstrated for a variety of cell-free expressed membrane proteins, including stearyl-CoA desaturase, glucan synthase, ATP synthase, DesK thermosensor, endothelin receptors $\mathrm{A}$ and $\mathrm{B}$, bacteriorhodopsin, connexin-43, aquaporin $\mathrm{Z}$, and the ion channels $\mathrm{Kcv}$ and KcsA. ${ }^{15-24}$ Given that incorporation of protein into the liposome cannot be facilitated by translocon components as these are not present in the lysate, it has been postulated that the presence of detergents, trace amounts of native lipids, or a close ribosome-liposome proximity aids protein insertion in the lipid bilayer of the liposomes. ${ }^{12-14}$

In principle, the bilayer self-insertion of cell-free expressed membrane proteins can be exploited as a purification method for ion channel electrophysiology, as demonstrated in a small number of recent studies. ${ }^{25-29}$ For example, large unilamellar 
vesicles containing the self-inserted ion channels VDAC or MscL have been purified from the expression mixture by density gradient centrifugation and subsequently fused with giant unilamellar vesicles (GUVs). The resulting proteoGUVs could be patch-clamped with planar aperture chips and single-channel current recordings of VDAC and MscL were obtained. ${ }^{25,26}$ Gradient-purified MscL-proteoliposomes have also been employed to deliver channels to an aperture-suspended bilayer, enabling single-channel MscL recordings with the BLM method. ${ }^{27}$ Interestingly, direct incorporation of membrane proteins in the 'measurement bilayer' has been achieved for expression mixture-exposed solid-supported bilayers, specifically for the odorant receptor OR5 and the transmembrane domain of the hERG potassium channel, enabling optical characterization of ligand binding without protein purification. ${ }^{28,29}$

Here, we expand the concept of direct bilayer incorporation of cell-free expressed membrane proteins to electrical characterization of ion channels in suspended lipid bilayers, as pioneered by Bayley and co-workers. ${ }^{23}$ The method employs a droplet-in-oil BLM electrophysiology configuration, and utilizes a cell-free reaction mixture rather than a dilute solution of purified proteoliposomes. The observation of single-channel current gating characteristic for KcsA, a proton-gated bacterial potassium channel, ${ }^{30}$ and for $\mathrm{hERG}_{\mathrm{S} 5-\mathrm{S} 6}$, the pore domain of a human cardiac potassium channel, ${ }^{31}$ demonstrates that functional cellfree expressed ion channels self-insert into suspended bilayers without the need for liposomes in the reaction mixture and without any purification step. This strategy hence combines the distinct advantages of in situ expression and plasma membrane incorporation of conventional patch clamp electrophysiology with the less complex instrumentation of the BLM technique, an assay format that is well suited to automation and scaling. ${ }^{7-9,32}$

The ion channels KcsA (wild-type and His-tagged) and a Histagged hERG construct with two transmembrane helices were expressed from plasmids with a commercial S30 lysate from $E$. coli and protein expression was verified by gel electrophoresis and Western blotting (see ESI $\dagger$ ). After expression, a $2 \mu \mathrm{L}$ droplet of the cell-free reaction mixture was placed at the tip of an agarcoated $\mathrm{Ag} / \mathrm{AgCl}$ electrode, which was subsequently immersed in a reservoir of decane oil with solubilized asolectin lipids, leading to the formation of a lipid monolayer at the aqueous-oil interface. A second $2 \mu \mathrm{L}$ droplet of pure buffer solution, typically $150 \mathrm{mM} \mathrm{KCl}, 10 \mathrm{mM}$ HEPES, pH 7.0 or pH 4.0, was introduced into the oil reservoir, in close vicinity to the reaction mixture droplet, at the tip of a second electrode. Next, the two droplets were brought into contact by manually manipulating the electrodes. This procedure leads to expulsion of the oil film at the contact area of the droplets, resulting in the formation of a stable lipid bilayer that separates the two aqueous volumes defined by the droplet-in-oil interiors, ${ }^{33,34}$ as schematically depicted in Fig. 1a. Bilayer formation is monitored by capacitance measurements, as outlined in the ESI. $\dagger$ It should be noted that the electrical properties of ion channels such as $\alpha$-hemolysin, OmpG, Kcv, KcsA, bacteriorhodopsin, gramicidin A and nystatin in interdroplet bilayers are indistinguishable from their properties in conventional aperture-suspended planar
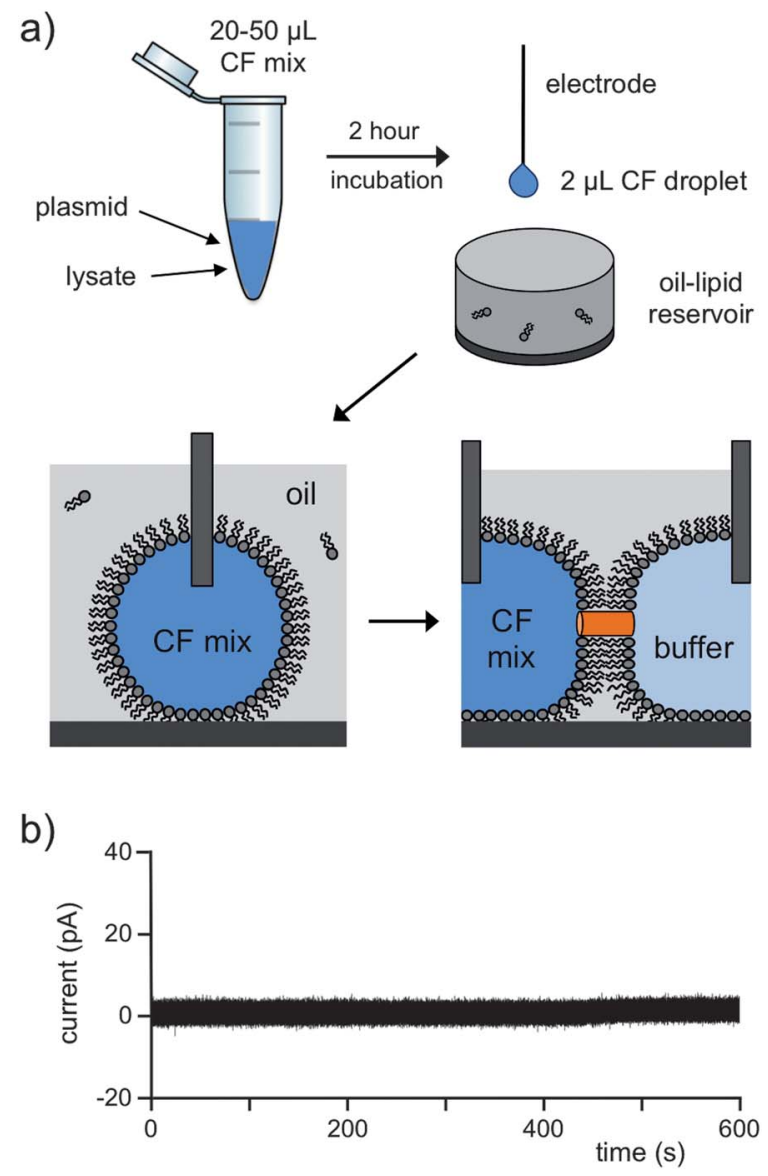

Fig. 1 Bilayer recordings of interdroplet bilayers exposed to a cell-free expression reaction mixture. (a) Schematic representation of interdroplet bilayer formation procedure. (b) Current trace of a lipid bilayer with a reaction mixture without plasmid in the first droplet and a buffer solution of $\mathrm{pH} 4$ in the second droplet.

bilayers (BLMs) ${ }^{33}$ which are attached to the wall of the aperture by an oil annulus.

An important advantage of the droplet-in-oil approach to BLM electrophysiology is that interdroplet bilayers are more stable than conventional aperture-suspended bilayers. ${ }^{33,34}$ However, cell-free expression mixtures are known to destabilize even interdroplet bilayers, which we have previously attributed to the high protein concentration in the lysate fraction, although the presence of other components such as PEG polymers, detergent molecules or residual E. coli lipids could also compromise the structural integrity of the bilayer. ${ }^{23,35}$ We previously reported that for $2 \mu \mathrm{L}$ droplets of a cell-free reaction mixture, bilayer lifetimes of at least 30 minutes could be obtained by a 10 -fold dilution of a liposome-supplemented mixture. ${ }^{35}$ However, in the present study we observed that also under these conditions the introduction of a buffer solution of $\mathrm{pH} 4$ as the second droplet, a requirement for proton-activated KcsA gating, resulted in unstable bilayers with an average lifetime of only $\sim 3$ minutes $(n=5)$. This was unexpected because interdroplet bilayers are known to be stable from $\mathrm{pH} 4$ to $\mathrm{pH} 10,{ }^{33}$ but this may be related to the relatively large size of our interdroplet bilayers, which are $\sim 250 \mu \mathrm{m}$ in diameter. However, by increasing the asolectin concentration in the decane 


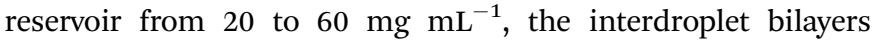
became sufficiently stable at $\mathrm{pH} 4$ to allow ion channel current measurements, without dilution of the reaction mixture and without liposome addition, as demonstrated by the bilayer current trace in Fig. 1b.

To establish whether KcsA is able to incorporate in an interdroplet bilayer from a cell-free reaction medium, a $2 \mu \mathrm{L}$ droplet of reaction medium ( $\mathrm{pH}$ 7.0) incubated with a plasmid encoding wild-type KcsA was introduced in the decane oil reservoir with 60 $\mathrm{mg} \mathrm{mL}^{-1}$ asolectin lipids. This KcsA reaction mixture droplet was then brought into contact with a droplet of $\mathrm{pH} 4$ buffer solution, which typically gave rise to a capacitance of $\sim 150-300 \mathrm{pF}$, indicative of the formation of an interdroplet bilayer with a diameter of $\sim 200-280 \mu \mathrm{m}$, assuming a specific bilayer capacitance of $0.5 \mu \mathrm{F} \mathrm{cm}^{-2} \cdot{ }^{34}$ Bilayer current traces were subsequently obtained at various voltage-clamp values. As shown in Fig. 2, at $+100 \mathrm{mV}$ a stable baseline current was obtained, with a peak-topeak noise of $\sim 5 \mathrm{pA}$ at $0.5 \mathrm{kHz}$ filtering, with occasional bursts of bilayer conductance, manifested as short-lived current steps with an amplitude of $11.5 \pm 2.2 \mathrm{pA}$, an average open-state dwell time of $4 \mathrm{~ms}$, and an intra-burst open probability of 0.19 . With $25 \mathrm{mM}$ or $50 \mathrm{mM}$ tetraethylammonium (TEA) in the droplet with the $\mathrm{pH}$ 4 solution, the average amplitude was reduced to $8.2 \pm 1.7 \mathrm{pA}$ and $6.2 \pm 1.1 \mathrm{pA}$, respectively (see $\mathrm{ESI}^{\dagger}$ ). The average current amplitude was measured at potentials between $+125 \mathrm{mV}$ and $-100 \mathrm{mV}$, giving larger current values at positive potentials than at the equivalent negative potentials (see ESI $\dagger$ ). Since the reaction

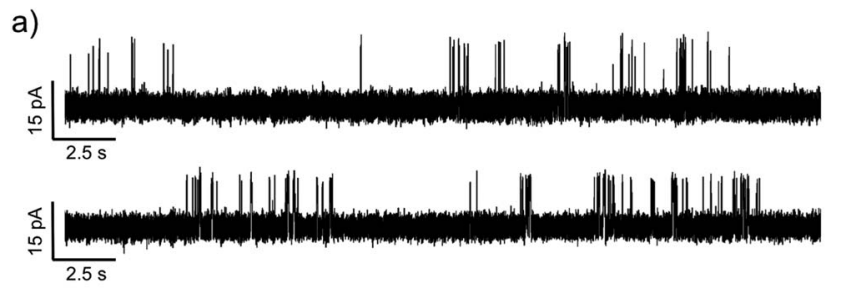

b)

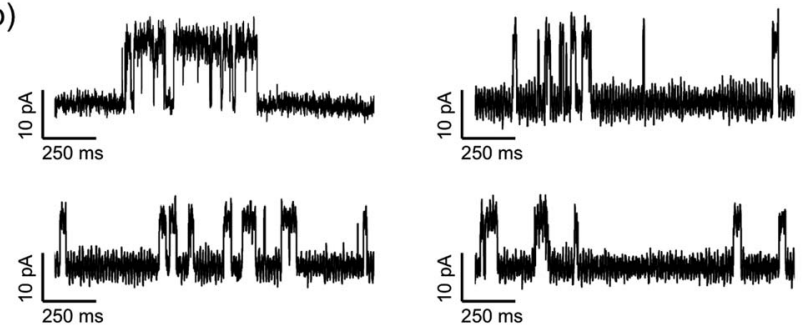

c)

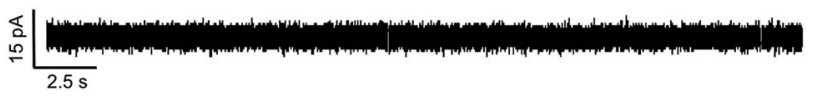

Fig. 2 Current traces of an interdroplet bilayer with a cell-free expression mixture after incubation of a plasmid encoding non-tagged KcsA as the first droplet and a buffer solution of $\mathrm{pH} 4$ (a-b) or $\mathrm{pH} 7$ (c) as the second droplet. (a) Overview of 30 second recordings at $+100 \mathrm{mV}$ (top row) and $+75 \mathrm{mV}$ (bottom row). (b) Zoomed-in view of two independent recordings at $+100 \mathrm{mV}$ (top row) and of two different regions of a single recording at $+75 \mathrm{mV}$ (bottom row). (c) Segment of a 1800 second trace recorded at $+100 \mathrm{mV}$ and $\mathrm{pH} 7$; no current steps are observed in the entire trace. medium without plasmid does not give rise to current events at pH 4 (Fig. 1b), the observed current activity can be attributed to an ion channel.

Notably, with a buffer solution of $\mathrm{pH} 7$ in the second droplet, these current events were not observed (Fig. 2), which is consistent with the known dependence of KcsA gating on pH. ${ }^{36,37}$ Moreover, the occurrence of current steps in discrete bursts with the characteristics described above corresponds with the literature on KcsA gating, particularly the 'low $\mathrm{P}_{\mathrm{o}}$ mode' identified by Chakrapani et al., who reported an open probability of 0.16 , an amplitude of $\sim 10 \mathrm{pA}$ (at $+100 \mathrm{mV}$ and $200 \mathrm{mM}$ $\mathrm{KCl}$ ), and an average open time of $\sim 5 \mathrm{~ms}$ in asolectin bilayers. ${ }^{37}$ Our results also agree with the gating characteristics of KcsA in bilayers of synthetic phospholipids with an asolectin-like intermediate content of anionic lipids. ${ }^{38}$ The mild rectification that is apparent from the obtained current-voltage curve and the $\sim 50 \%$ reduction in the amplitude of the current step by the potassium channel blocker tetraethylammonium (see $\mathrm{ESI} \dagger$ ) is also in agreement with previous studies on KcsA. ${ }^{38-40}$ Although a full characterization would require measurements with other cations than potassium to verify the permeability profile of $\mathrm{KcsA},{ }^{40}$ we conclude that the current steps measured at $\mathrm{pH} 4$ (Fig. 2) correspond to KcsA ion channels that have inserted into the interdroplet bilayer from the reaction mixture.

Next, we investigated an ion channel construct of which single-channel electrophysiology data has, to our knowledge, not previously been obtained: the pore domain of the human Kv11.1 potassium channel (hERG), which comprises the porelining helices S5 and S6 and the connecting pore loop. ${ }^{31}$ A cellfree reaction mixture was placed as a droplet-in-oil in the decane-asolectin reservoir after incubation with a hERG ${ }_{\mathrm{S} 5-\mathrm{S} 6}$ encoding plasmid, and a bilayer was formed between the hERG $_{\text {S5-S6 }}$ expression droplet and a droplet with $\mathrm{pH} 7$ buffer solution. Long bursts of current steps, shown in Fig. 3, were readily observed at $+100 \mathrm{mV}$, typically, as with the KcsA experiments described above, 1-2 minutes after formation of the interdroplet bilayer. We identified two populations, a largeconductance opening with an amplitude of $24.3 \pm 2.9 \mathrm{pA}$, a mean open lifetime of $1 \mathrm{~ms}$, and an open probability of 0.07 , and a small-conductance opening of $3.7 \pm 0.4 \mathrm{pA}$, a mean open time of $0.3 \mathrm{~ms}$, and an open probability of 0.03 . We attribute these current events to hERG ion channel gating because of a close resemblance to gating characteristics of purified liposome-reconstituted hERG $_{\mathrm{S} 5-\mathrm{S} 6}$ delivered to conventional aperture-suspended bilayers (unpublished data).

As transitions between the two conductance states typically do not occur in a single experiment, we speculate that the low and the high conductance current events result from different conformations of the bilayer-incorporated hERG $_{\mathrm{S} 5-\mathrm{S} 6}$ pore domain, which in the absence of the flanking voltage sensor and C-terminal domains may not have a single stable open state. It should be noted that the small number of single-channel patch clamp studies of full-length (voltage-gated) hERG suggest a conductance of approximately $10 \mathrm{pS}$, for example a current amplitude of $0.3 \mathrm{pA}$ at $-40 \mathrm{mV}$ and $100 \mathrm{mM} \mathrm{KCl}$ with an average open time of $3.2 \mathrm{~ms}$, or an amplitude of $\sim 1 \mathrm{pA}$ at $-120 \mathrm{mV} .^{31,41}$ Hence the observed low conductance state of the $\mathrm{hERG}_{\mathrm{S} 5-\mathrm{S} 6}$ pore 
a)

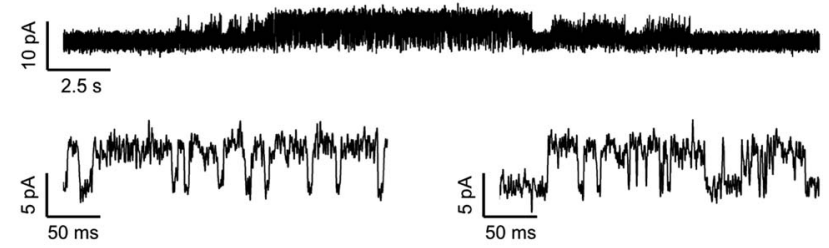

b)
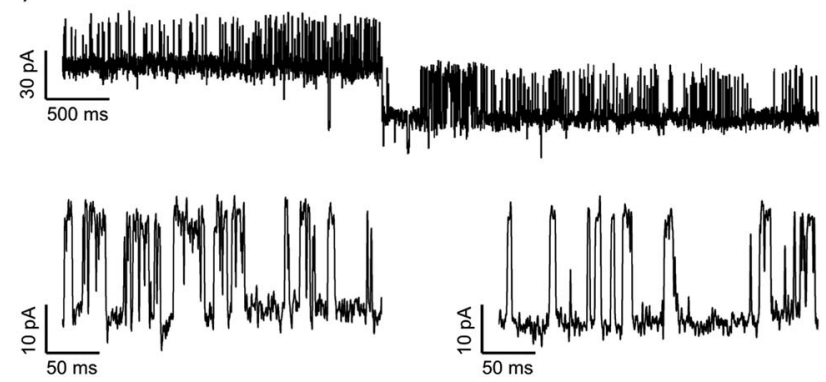

Fig. 3 Current traces of an interdroplet bilayer with a cell-free expression mixture after incubation of a plasmid encoding $\mathrm{hERG}_{\mathbf{S 5}-{ }_{56}}$ as the first droplet and a buffer solution of $\mathrm{pH} 7$ as the second droplet. Overview and zoomed-in traces recorded at $+100 \mathrm{mV}$ depict (a) small-conductance current events, and (b) largeconductance current events from independent experiments.

domain is most likely more relevant than the large conductance state. However, in the context of the present study, the pertinent point is that the bilayer current events in the hERG $_{\text {S5-S6 }}$ system are distinctly different from those in the KcsA system.

These experiments with non-tagged KcsA and the His-tagged hERG pore domain validate the concept that bilayer insertion from a cell-free reaction mixture allows single-channel electrophysiological measurements to be made on expressed ion channels, analogous to the optical characterization of drug binding to membrane proteins that self-insert in solid-supported bilayers. ${ }^{28,29}$ Bayley and co-workers previously reported that the interaction of the small potassium channel Kcv with a series of channel blockers could be investigated at the singlechannel level after introduction of a liposome-supplemented cell-free reaction mixture in a lipid-free oil phase, followed by droplet contact with a series of lipid-coated droplets containing different drug solutions. ${ }^{23}$ However, we observed that although addition of liposomes to the reaction mixture results in a higher yield of protein expression (see ESI $\dagger$ ), it becomes more difficult to obtain single-channel recordings. Typically, interdroplet bilayer formation with liposome-supplemented reaction mixture droplets gives rise to fluctuating elevated current baselines of $>100 \mathrm{pA}$ and bilayer failure (data not shown). We hypothesize that proteoliposomes from the reaction mixture fuse with the interdroplet bilayer, either causing membrane destabilization by incorporation of an excess amount of the expressed ion channel, or by delivering bilayer-disrupting molecules that have adsorbed on the liposome surface during the transcription-translation incubation period.

In summary, direct incorporation in suspended lipid bilayers of ion channels produced by in vitro transcription-translation, which is expected to be feasible for all ion channels that are known to self-insert into liposomes from a cell-free reaction medium, ${ }^{\mathbf{1 2 - 1 4}}$ enables single-channel characterization of ion channels without the need for cell culture and protein purification steps. Implementation as droplet-in-oil BLM electrophysiology is extremely favorable because only microliter volumes of the relatively expensive commercial cell lysates are required. Moreover, this method could take advantage of the recent achievements in parallel BLM arrays, ${ }^{7,42,43}$ particularly in combination with miniaturized amplifier electronics, ${ }^{\mathbf{4 4 , 4 5}}$ to achieve more cost-effective and smaller-footprint electrophysiological drug discovery or drug safety screening of biomedically important ion channels such as the full-length hERG cardiac potassium channel.

\section{Acknowledgements}

The authors would like to thank Anthony G. Lee (University of Southampton) for advice and support, Alvin Shrier (McGill University) for kindly providing the full-length hERG plasmid, and Andrée E. Gravel under the supervision of Jason C. Young (McGill University) for preparing the hERG $_{\mathrm{S} 5-\mathrm{S} 6}$ plasmid. M. P. gratefully acknowledges funding from the Engineering and Physical Sciences Research Council (EPSRC grant EP/I029036/1) and I. M. from the Natural Sciences and Engineering Research Council (NSERC) of Canada. M. B. wishes to thank the Faculté des Sciences of the Université du Québec à Montréal, the Ministère de l'Éducation, du Loisir et du Sport du Québec, the NSERC Bionanomachines training program, the Centre Québécois sur les Matériaux Fonctionnels (CQMF) and Fonds de Recherche du Québec - Nature et Technologies (FRQNT) for the award of scholarships. I. M. is a member of the CQMF and the Groupe de recherche axé sur la structure des protéines (GRASP).

\section{References}

1 J. P. Overington, B. Al-Lazikani and A. L. Hopkins, Nat. Rev. Drug Discovery, 2006, 5, 993-996.

2 B. Hille, Ion Channels of Excitable Membranes, Sinauer Associates, Sunderland, 3rd edn, 2001.

3 S. Demarche, K. Sugihara, T. Zambelli, L. Tiefenauer and J. Vörös, Analyst, 2011, 136, 1077-1089.

4 P. Kongsuphol, K. B. Fang and Z. Ding, Sens. Actuators, B, 2013, 185, 530-542.

5 J. Dunlop, M. Bowlby, R. Peri, D. Vasilyev and R. Arias, Nat. Rev. Drug Discovery, 2008, 7, 358-368.

6 S. Stoelzle, A. Obergrussberger, A. Brüggemann, C. Haarmann, M. George, R. Kettenhofen and N. Fertig, Front. Pharmacol., 2011, 2, 76.

7 M. Zagnoni, Lab Chip, 2012, 12, 1026-1039.

8 T. Thapliyal, J. L. Poulos and J. J. Schmidt, Biosens. Bioelectron., 2011, 26, 2651-2654.

9 G. Baaken, M. Sondermann, C. Schlemmer, J. Rühe and J. C. Behrends, Lab Chip, 2008, 8, 938-944.

10 M. Kreir, C. Farre, M. Beckler, M. George and N. Fertig, Lab Chip, 2008, 8, 587-595.

11 J. W. Whittaker, Biotechnol. Lett., 2013, 35, 143-152.

12 F. Katzen, T. C. Petersen and W. Kudlicki, Trends Biotechnol., 2009, 27, 455-460. 
13 F. Junge, S. Haberstock, C. Roos, S. Stefer, D. Proverbio, V. Dötsch and F. Bernhard, New Biotechnol., 2011, 28, 262-271.

14 C. Roos, L. Kai, D. Proverbio, U. Ghoshdastider, S. Filipek, V. Dötsch and F. Bernard, Mol. Membr. Biol., 2013, 30, 75-89.

15 M. A. Goren and B. G. Fox, Protein Expression Purif., 2008, 62, 171-178.

16 A. Periasamy, N. Shadiac, A. Amalraj, S. Garajová, Y. Nagarajan, S. Waters, H. D. Mertens and M. Hrmova, Biochim. Biophys. Acta, 2013, 1828, 743-757.

17 D. Matthies, S. Haberstock, F. Joos, V. Dötsch, J. Vonck, F. Bernhard and T. Meier, J. Mol. Biol., 2011, 413, 593-603.

18 M. Martín, D. Albanesi, P. M. Alzari and D. de Mendoza, Protein Expression Purif., 2009, 66, 39-45.

19 D. Proverbio, C. Roos, M. Beyermann, E. Orbán, V. Dötsch and F. Bernhard, Biochim. Biophys. Acta, 2013, 1828, 21822192.

20 R. Kalmbach, I. Chizhov, M. C. Schumacher, T. Friedrich, E. Bamberg and M. Engelhard, J. Mol. Biol., 2007, 371, 639-648.

21 Y. Moritani, S. M. Nomura, I. Morita and K. Akiyoshi, FEBS J., 2010, 277, 3343-3352.

22 N. T. Hovijitra, J. J. Wuu, B. Peaker and J. R. Swartz, Biotechnol.Bioeng., 2009, 104, 40-49.

23 R. Syeda, M. A. Holden, W. L. Hwang and H. Bayley, J. Am. Chem. Soc., 2008, 130, 15543-15548.

24 A. van Dalen, M. van der Laan, A. J. Driessen, J. A. Killian and B. de Kruijff, FEBS Lett., 2002, 511, 51-58.

25 A. Varnier, F. Kermarrec, I. Blesneac, C. Moreau, L. Liguori, J. L. Lenormand and N. Picollet-D'hahan, J. Membr. Biol., 2010, 233, 85-92.

26 C. Berrier, I. Guilvout, N. Bayan, K. H. Park, A. Mesneau, M. Chami, A. P. Pugsley and A. Ghazi, Biochim. Biophys. Acta, 2011, 1808, 41-46.

27 C. E. Price, A. Kocer, S. Kol, J. P. van der Berg and A. J. M. Driessen, FEBS Lett., 2011, 585, 249-254.

28 R. Robelek, E. S. Lemker, B. Wiltschi, V. Kirste, R. Naumann, D. Oesterhelt and E. K. Sinner, Angew. Chem., Int. Ed., 2007, 46, 605-608.
29 A. A. Yildiz, C. Kang and E. K. Sinner, Analyst, 2013, 138, 2007-2012.

30 S. Uysal, L. G. Cuello, D. M. Cortes, S. Koide, A. A. Kossiakoff and E. Perozo, Proc. Natl. Acad. Sci. U. S. A., 2011, 108, 1189611899.

31 J. I. Vandenberg, M. D. Perry, M. J. Perrin, S. A. Mann, Y. Ke and A. P. Hill, Physiol. Rev., 2012, 92, 1393-1478.

32 M. Zagnoni, M. E. Sandison and H. Morgan, Biosens. Bioelectron., 2009, 24, 1235-1240.

33 H. Bayley, B. Cronin, A. Heron, M. A. Holden, W. L. Hwang, R. Syeda, J. Thompson and M. Wallace, Mol. BioSyst., 2008, 4, 1191-1208.

34 S. Aghdaei, M. E. Sandison, M. Zagnoni, N. G. Green and H. Morgan, Lab Chip, 2008, 8, 1617-1620.

35 M. S. Friddin, H. Morgan and M. R. R. de Planque, Biomicrofluidics, 2013, 7, 014108.

36 L. G. Cuello, J. G. Romero, D. M. Cortes and E. Perozo, Biochemistry, 1998, 37, 3229-3236.

37 S. Chakrapani, J. F. Cordero-Morales and E. Perozo, J. Gen. Physiol., 2007, 130, 479-496.

38 P. Marius, M. Zagnoni, M. E. Sandison, J. M. East, H. Morgan and A. G. Lee, Biophys. J., 2008, 94, 1689-1698.

39 E. Kutluay, B. Roux and L. Heginbotham, Biophys. J., 2005, 88, 1018-1029.

40 M. LeMasurier, L. Heginbotham and C. Miller, J. Gen. Physiol., 2001, 118, 303-313.

41 J. Kiehn, A. E. Lacerda and A. M. Brown, Am. J. Physiol., 1999, 277, H199-H210.

42 J. L. Poulos, T. J. Jeon, R. Damoiseaux, E. J. Gillespie, K. A. Bradley and J. J. Schmidt, Biosens. Bioelectron., 2009, 24, 1806-1810.

43 R. Kawano, Y. Tsuji, K. Sato, T. Osaki, K. Kamiya, M. Hirano, T. Ide, N. Miki and S. Takeuchi, Sci. Rep., 2013, 3, 1995.

44 F. Thei, M. Rossi, M. Bennati, M. Crescentini, F. Lodesani, H. Morgan and M. Tartagni, IEEE Trans. Nanotechnol., 2010, 9, 295-302.

45 J. K. Rosenstein, S. Ramakrishnan, J. Roseman and K. L. Shepard, Nano Lett., 2013, 13, 2682-2686. 